\title{
AN EXACT SEQUENCE IN GALOIS COHOMOLOGY
}

\author{
DOCK SANG RIM ${ }^{1}$
}

Let $A$ be an integrally closed noetherian domain with the quotient field $F$. The group of divisors of $A$ is the free abelian group generated by nonzero minimal prime ideals of $A$ and is denoted by $D(A)$. This is canonically isomorphic to the group gotten from the set of all reflexive $A$-ideals (including fractional ideals) under the rule $\mathfrak{a} \cdot \mathfrak{b}$ $=(\mathfrak{a} \cdot \mathfrak{b})^{* *}$ where $\mathfrak{c}^{*}=\{a \in F \mid a \mathfrak{c} C A\}=\operatorname{Hom}_{A}(\mathfrak{c}, A)$. The divisor class group of $A$ denoted by $C(A)$ is the factor group of $D(A)$ by the principal divisors, i.e. it is defined by the exact sequence ${ }^{2}$

$$
0 \rightarrow U(F) / U(A) \stackrel{D}{\rightarrow} D(A) \rightarrow C(A) \rightarrow 0
$$

where $D(a)=\sum_{\mathfrak{p}} \nu_{\mathfrak{p}}(a) \mathfrak{p}$ with $\left(\mathfrak{p} A_{\mathfrak{p}}\right)_{\mathfrak{p}}(a)=a A_{\mathfrak{p}}$. We observe that $A$ is a unique factorization domain if and only if $C(A)=0$, i.e. if and only if $U(F) / U(A) \rightarrow D(A)$ is an isomorphism.

Now let $S \supset R$ be an integral extension of an integrally closed noetherian domain, whose quotient field $L \supset K$ is a separable extension of finite degree. Then we obtain the canonical map $i: D(R) \rightarrow D(S)$ given by $\sum_{\mathfrak{p}} \nu_{\mathfrak{p}} \mathfrak{p} \rightarrow \sum_{\mathfrak{p}} \nu_{\mathfrak{p}}\left(\sum_{P \mid \mathfrak{p}} e(\mathfrak{B}) \mathfrak{B}\right)$ where $e(\mathfrak{B})$ is the ramification index of $\mathfrak{B}$ in $S \supset R$, i.e. $\mathfrak{p} S_{\mathfrak{P}}=\left(\mathfrak{P} S_{\mathfrak{P}}\right)^{e(\mathfrak{B})}$. Since the map $i$ sends the principal divisors to principal divisors, it induces the map $i: C(R)$ $\rightarrow C(S)$. We denote the kernel of $i$ by $C(S / R)$. Thus $C(S / R)$ is the subgroup of $C(R)$ consisting of those divisor classes which become principal under the extension $S \supset R$. Now let the quotient field extension $L \supset K$ be Galois with the Galois group $G$. As customary we denote $H^{n}(G, U(L)), H^{n}(G, U(S))$ by $H^{n}(L / K), H^{n}(S / R)$ respectively. The main purpose of this short note is to prove:

THEOREM. Let $S \supset R$ be an integral extension of an integrally closed noetherian domain whose quotient field extension $L \supset K$ is Galois with the Galois group $G$. Then we have the exact sequence

$$
\begin{aligned}
0 & \rightarrow C(S / R) \rightarrow H^{1}(S / R) \rightarrow D(S)^{G} / i D(R) \rightarrow C(S)^{G} / i C(R) \rightarrow \\
& \rightarrow H^{2}(S / R) \rightarrow \bigcap_{\mathfrak{p}} H^{2}\left(S_{\mathfrak{p}} / R_{\mathfrak{p}}\right) \rightarrow H^{1}(G, C(S)) \rightarrow H^{3}(S / R)
\end{aligned}
$$

Received by the editors May 14, 1964.

${ }^{1}$ This work was partially supported by NSF GP-218.

2 For any commutative ring $A$, we denote by $U(A)$ the group of invertible elements in $A$. 
where $\bigcap_{\mathfrak{p}} H^{2}\left(S_{\mathfrak{p}} / R_{\mathfrak{p}}\right)=\bigcap_{\mathfrak{p}} \operatorname{Im}\left(H^{2}\left(S_{\mathfrak{p}} / R_{\mathfrak{p}}\right) \rightarrow H^{2}(L / K)\right)$, p running through all nonzero minimal primes of $R$.

REMARK. A somewhat similar exact sequence related to the Brauer groups in the case when $S \supset R$ is unramified was obtained in [2], [3].

Proof. Firstly we observe that $H^{1}(G, D(S))=0$. Indeed, if we fix, for each nonzero minimal prime $\mathfrak{p}$ in $R$, a nonzero minimal prime $\mathfrak{P}$ in $S$ lying above $\mathfrak{p}$, and if we denote by $G_{\mathfrak{p}}$ the decomposition subgroup of $\mathfrak{B}$ over $\mathfrak{p}$, then $D(S) \cong \sum_{\mathfrak{p}} Z\left[G_{\mathfrak{p}}\right] \otimes_{z[G]} Z$ as $G$-modules, where $\mathfrak{p}$ runs through all nonzero minimal primes of $R$. Consequently $H^{*}(G, D(S))=\sum_{\mathfrak{p}} H^{*}\left(G_{\mathfrak{p}}, Z\right)$ and in particular we have $H^{1}(G, D(S))$ $=0$. Now for each minimal prime $\mathfrak{p}$ in $R, S_{\mathfrak{p}}$ is a unique factorization domain and hence $0 \rightarrow U\left(S_{\mathfrak{p}}\right) \rightarrow U(L) \rightarrow D\left(S_{\mathfrak{p}}\right) \rightarrow 0$ is an exact sequence of $G$-modules. Therefore $0 \rightarrow H^{2}\left(S_{\mathfrak{p}} / R_{\mathfrak{p}}\right) \rightarrow H^{2}(L / K) \rightarrow H^{2}\left(G, D\left(S_{\mathfrak{p}}\right)\right)$ is exact and hence we obtain the exact sequence

$$
0 \rightarrow \bigcap_{\mathfrak{p}} H^{2}\left(S_{\mathfrak{p}} / R_{\mathfrak{p}}\right) \rightarrow H^{2}(L / K) \rightarrow H^{2}(G, D(S)) .
$$

The exact sequence of $G$-modules $0 \rightarrow U(L) / U(S) \rightarrow D(S) \rightarrow C(S) \rightarrow 0$ together with $H^{1}(G, D(S))=0$ gives us the exact sequences

$$
\begin{gathered}
0 \rightarrow(U(L) / U(S))^{G} \rightarrow D(S)^{G} \rightarrow C(S)^{G} \rightarrow H^{1}(G, U(L) / U(S)) \rightarrow 0, \\
0 \rightarrow H^{1}(G, C(S)) \rightarrow H^{2}(G, U(L) / U(S)) \rightarrow H^{2}(G, D(S)) .
\end{gathered}
$$

In turn the exact commutative diagram

$$
\begin{aligned}
& \begin{array}{ccc}
0 \rightarrow U(K) / U(R) & \rightarrow & D(R) \rightarrow C(R) \rightarrow 0 \\
\downarrow & \downarrow & \downarrow
\end{array} \\
& 0 \rightarrow(U(L) / U(S))^{G} \rightarrow D(S)^{G} \rightarrow C(S)^{G} \rightarrow H^{1}(G, U(L) / U(S)) \rightarrow 0
\end{aligned}
$$

yields the exact sequence

$$
0 \rightarrow C(S / R) \rightarrow(U(L) / U(S))^{G} /(U(K) / U(R)) \rightarrow D(S)^{G / i D}(R)
$$

$$
\rightarrow C(S)^{a} / i C(R) \rightarrow H^{1}(G, U(L) / U(S)) \rightarrow 0 .
$$

On the other hand, the exact sequence $0 \rightarrow U(S) \rightarrow U(L) \rightarrow U(L) / U(S)$ $\rightarrow 0$ together with Hilbert's Theorem 90 gives us the exact sequences

$$
\begin{aligned}
& 0 \rightarrow U(R) \rightarrow U(K) \rightarrow(U(L) / D(S))^{G} \rightarrow H^{1}(S / R) \rightarrow 0 \\
& 0 \rightarrow H^{1}(G, U(L) / U(S)) \rightarrow H^{2}(S / R) \rightarrow H^{2}(L / K) \\
& \rightarrow H^{2}(G, U(L) / U(S)) \rightarrow H^{3}(S / R) \rightarrow \cdots
\end{aligned}
$$

Now the exact commutative diagram 
$0 \rightarrow H^{1}(G, U(L) / U(S)) \rightarrow$

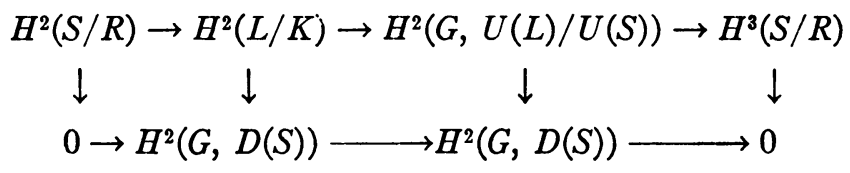

together with (1) and (3) yields the exact sequence

$$
\begin{gathered}
0 \rightarrow H^{1}(G, U(L) / U(S)) \rightarrow H^{2}(S / R) \rightarrow \bigcap_{\mathfrak{p}} H^{2}\left(S_{\mathfrak{p}} / R_{\mathfrak{p}}\right) \\
\rightarrow H^{1}(G, C(S)) \rightarrow H^{3}(S / R) .
\end{gathered}
$$

On the other hand, (4) and (5) gives us

$$
\begin{aligned}
0 \rightarrow C(S / R) \rightarrow & H^{1}(S / R) \rightarrow D(S)^{G} / i D(R) \rightarrow C(S)^{G} / i C(R) \\
& \rightarrow H^{1}(G, U(L) / U(S)) \rightarrow 0 .
\end{aligned}
$$

Connecting (7) and (8) we obtain the desired exact sequence.

When $S \supset R$ is unramified, we can relate the 2-dimensional cohomology group with the Brauer group. We denote by $B(S / R)$ the kernel of the canonical map $B(R) \rightarrow B(S)$, where $B($ ) denotes the Brauer group. Then $H^{2}(S / R)=B(S / R)$ if $S \supset R$ is unramified and $R$ is a local domain [1] and thus we obtain:

CoROllary 1. Let $S \supset R$ be unramified. Then we have the exact sequence

$$
\begin{aligned}
0 \rightarrow H^{1}(S / R) \rightarrow & C(R) \rightarrow C(S)^{G} \rightarrow H^{2}(S / R) \rightarrow \bigcap_{\mathfrak{p}} B\left(S_{\mathfrak{p}} / R_{\mathfrak{p}}\right) \\
& \rightarrow H^{1}(G, C(S)) \rightarrow H^{3}(S / R) .
\end{aligned}
$$

PRoof. If $S \supset R$ is unramified, then $D(S)^{G}=i D(R)$ and $\cap_{\mathfrak{p}} H^{2}\left(S_{\mathfrak{p}} / R_{\mathfrak{p}}\right)$ $=\bigcap_{\mathfrak{p}} B\left(S_{\mathfrak{p}} / R_{\mathfrak{p}}\right)$.

If we further assume $R$ to be regular, our exact sequence coincides with the exact sequence in [2], [3].

CoRollary 2. Let $S \supset R$ be unramified. If $R$ is regular, we have the exact sequence

$$
\begin{aligned}
0 \rightarrow H^{1}(S / R) & \rightarrow C(R) \rightarrow C(S)^{G} \rightarrow H^{2}(S / R) \rightarrow B(S / R) \\
& \rightarrow H^{1}(G, C(S)) \rightarrow H^{3}(S / R) .
\end{aligned}
$$

Proof. We must show that $\bigcap_{\mathfrak{p}} B\left(S_{\mathfrak{p}} / R_{\mathfrak{p}}\right)=B(S / R)$. Since one side inclusion is clear [1], it suffices to show that $\bigcap_{\mathfrak{p}} B\left(S_{\mathfrak{p}} / R_{\mathfrak{p}}\right) \subset B(S / R)$, i.e. $\operatorname{Ker}\left(H^{2}(L / K) \rightarrow H^{2}(G, D(S)) \subset B(S / R)\right.$. Now $S$, being unramified over a regular domain $R$, is also regular and hence is a local unique factorization domain. Consequently $D(S)$ is nothing but the group of 
invertible $S$-ideals. Let $\alpha \in \operatorname{Ker}\left(H^{2}(L / K) \rightarrow H^{2}(G, D(S))\right)$, and let $\left\{a_{\sigma, \tau}\right\}$ be a 2-cocycle representing $\alpha$. This means that there exists a set $\left\{A_{\sigma}\right\}$ of invertible $S$-ideals indexed by $G$ such that $a_{\sigma, \tau} A_{\sigma} A_{\tau}^{\sigma} A_{\sigma \tau}^{-1}$ $=S$, i.e. $a_{\sigma, \tau} A_{\sigma} A_{\tau}^{\sigma}=A_{\sigma \tau}$. (We may assume that $A_{1}=S$.) Now let $\Gamma$ be the central simple $K$-algebra associated with the 2-cocycle $\left\{a_{\sigma, \tau}\right\}$, i.e. $\Gamma=\sum_{\sigma} L u_{\sigma}$ (direct sum) with the multiplication rule: $\left(x_{\sigma} u_{\sigma}\right)\left(x_{\tau} u_{\tau}\right)$ $=x_{\sigma} x_{\tau}^{\sigma} a_{\sigma, \tau} u_{\sigma \tau}$. Then $\Lambda=\sum_{\sigma} A_{\sigma} u_{\sigma}$ which is a subset of $\Gamma$ is stable under the multiplication since $A_{\sigma} u_{\sigma} A_{\tau} u_{\tau}=A_{\sigma} A_{\tau}^{\sigma} a_{\sigma, \tau} u_{\sigma \tau}=A_{\sigma \tau} u_{\sigma \tau}$. Thus $\Lambda$ is an order over $R$ in $\Gamma$, and is projective as an $R$-module. Now consider the canonical map $S \otimes_{R} \Lambda \rightarrow \operatorname{Hom}_{S}(\Lambda, \Lambda)$ given by $(s \otimes \lambda)(x)=s \chi \lambda$. Since $\alpha \in \operatorname{Ker}\left(H^{2}(L / K) \rightarrow H^{2}(G, D(S))=\bigcap_{\mathfrak{p}} H^{2}\left(S_{\mathfrak{p}} / R_{\mathfrak{p}}\right)\right.$, it follows that $S_{\mathfrak{p}} \otimes \Lambda_{\mathfrak{p}} \rightarrow \operatorname{Hom}_{S \mathfrak{p}}\left(\Lambda_{\mathfrak{p}}, \Lambda_{\mathfrak{p}}\right)$ is an isomorphism for all non-zero minimal primes $\mathfrak{p}$ in $R$. Consequently the canonical map $S \otimes_{R} \Lambda \rightarrow \operatorname{Hom}_{S}(\Lambda, \Lambda)$ is an isomorphism since both sides are $R$-projective modules of the same rank. Therefore $\Lambda$ is an $R$-separable order in $\Sigma$ with $S$ as a splitting ring, and this completes our proof.

\section{BIBLIOGRAPHY}

1. M. Auslander and O. Goldman, The Brauer group of a commutative ring, Trans. Amer. Math. Soc. 97 (1960), 367-409.

2. M. Auslander and A. Brumer, The Brauer group and Galois cohomology of commutative rings, Trans. Amer. Math. Soc. (to appear)

3. S. Chase, D. Harrison and A. Rosenberg, Galois theory and Galois cohomology of commutative rings, Memoirs Amer. Math. Soc., no. 52, 1965.

BRANDEIS UNIVERSITY 